\title{
IMMUNOLOGICAL STUDIES ON THE INFLUENCES OF ISOLATION PROCEDURES UPON CONTENTS OF URINARY GLYCOPROTEIN AND GLYCOSAMINOGLYCANS
}

\author{
HIROSHI MASUDA, SHIGEKI SHICHIJO \\ AND MUTSUYA TAKEUCHI \\ Institute of Clinical Pathology, Kurume University \\ School of Medicine, Kurume, 830, Japan
}

Received for publication October 19, 1978

\begin{abstract}
A study has been made for the purpose of methodological aspect on Tamm-Horsfall glycoprotein, glycosaminoglycans and glycopeptide contents in such urinary fractions as the one the filtered and the other the supernatant following centrifugation. The contents were measured both biochemically and immunologically. The filtration procedure greatly reduced the Tamm-Horsfall glycoprotein content, while the centrifugation had little effect on its content. Furthermore, either filtration procedure or centrifugation had no effect on the any of uronic acid, hexose or sialic acid content of the glycosaminoglycan-glycopeptide preparations.
\end{abstract}

\section{INTRODUCTION}

Elevations of the urinary TammHorsfall glycoprotein level in patients with diseases of the kidney and urinary tract were reported (Saito, 1973), while the significance of the elevated urinary Tamm-Horsfall glycoprotein level in these patients is still unknown. On the other hand, consistent increase of urinary glycosaminoglycans in patients with hereditary mucopolysaccharidoses has been described by numerous investigators. Recently, the changes of urinary glycosaminoglycans in diseases have been reviewed (McKusick, 1972). This suggested more studies on the complex carbohydrate content in different fractions of these isolation processes from the urine should be added to this field. It is the purpose of this paper to com- pare the distribution of Tamm-Horsfall glycoprotein, glycosaminoglycans and glycopeptide in different fractions of these isolation procedures from the urine.

\section{MATERIALS AND METHODS}

\section{Materials}

Tamm-Horsfall glycoprotein, glycosaminoglycans and glycopeptide were prepared from normal human urines, collected for 24 hours from five male subjects between the aged of 30 and 40 years.

\section{Preparation of urinary fractions}

One volume of urine samples was added with equal volume of deionized 
water and then was divided into two groups. The one was filtered with Toyo filter paper No. 2 (Japan) and the filtered fraction was saved for further treatment. The other was centrifuged at 1,500 r.p.m. for 15 minutes and the supernatant fraction was also saved for further treatment.

\section{Isolation of Tamm-Horsfall glycopro- tein}

Tamm-Horsfall glycoprotein was isolated by the method of Tamm-Horsfall (1950) from each of three urine fractions mentioned above. Solid $\mathrm{NaCl}$ was added to the urine fractions to a concentration of $0.58 \mathrm{M}$. Mixture was left overnight at $4^{\circ} \mathrm{C}$, then centrifuged at 4,000 r.p.m. for 30 minutes. After washing with $0.58 \mathrm{M} \mathrm{NaCl}$, the precipitate was resuspended in deionized water, and then used both for chemical analyses and the Ouchterlony double diffusion test.

\section{Isolation of glycosaminoglycans and} glycopeptide

Glycosaminoglycans and glycopeptide were isolated from each of three urine fractions which were mentioned before according to the procedure of Schiller et al. (1961). The urine fractions were digested with papain (EC 3.4.10) in the presence of EDTA and cysteine at $65^{\circ} \mathrm{C}$ for 48 hours at $\mathrm{pH} 6.4$. The mixture was adjusted to $\mathrm{pH} 7.8$ and then digested with trypsin (EC 3.4.4.4) at $37^{\circ} \mathrm{C}$ for 72 hours with simultaneous dialysis against $1 / 15 \mathrm{M}$ phosphate buffer, $\mathrm{pH}$ 7.8. The digested solution was deproteinized with $5 \%$ trichloroacetic acid in the cold, and then solution was dialyzed against several changes of cold deionized water for 72 hours. Insoluble material was centrifuged out and the supernatant (the glycosaminoglycans and glycopeptide preparation) was freeze-dried. The preparation was dissolved in a small amount of deionized water, and was utilized both for chemical analyses and cellulose acetate electrophoresis.

\section{Analytical procedures}

Protein content was estimated by the Lowry's method, with human serum (Hyland Laboratory, U.S. A.) as a standard (Lowry, 1951). Hexose content was estimated by orcinol-sulfuric acid method of Winzler, utilizing a mixture of mannose and galactose as a standard (Winzler, 1955a) and sialic acid content by Winzler's diphenylamine method, using $\mathrm{N}$-acetylneuraminic acid as a standard (Winzler, 1955b), respectively. Uronic acid content was determined by carbazole reaction of Dische, with glucuronic acid as a standard (Dische, 1962).

\section{Immunological studies}

Rabbits, weighting appr ximately 3 $\mathrm{kg}$, were immunized by four footpad injections of isolated Tamm-Horsfall glycoprotein (20 $\mathrm{mg}$ in each injestion) which had previously been emulsified by adding the complete Freund's adjuvant over 5 weeks. Against the obtained antiserum, the antigenic activity of the isolated Tamm-Horsfall glycoprotein was examined by the Ouchterlony double diffusion test and immunoelectrophoresis as employed in the routine works.

\section{Electrophoresis of glycosaminoglycans on a cellulose acetate strip}

The material was run in a horizontal type apparatus at constant current of $1.0 \mathrm{~mA} / \mathrm{cm}$ on an OXOID strip $(8 \times 1.2$ $\mathrm{cm}$ ) using both barbital buffer, $\mathrm{pH}$ 8.6, $\mu=0.06$ for 28 minutes and $0.3 \mathrm{M}$ cal- 
cium acetate, pH 7.5 for 3 hours. 5 to $10 \mu \mathrm{l}$ of aqueous suspensions of preparations $10 \mathrm{mg}$ per $0.1 \mathrm{ml}$ were used in this study. After electrophoresis, the strip was stained with alcian blue in the normal way with chondroitin-4-sulfate, chondroitin-6-sulfate, dermatan sulfate, heparan sulfate and hyaluronic acid as standards; all of them were purchased from Seikagaku Kogyo Co., Ltd. (Tokyo, Japan). Both chondroitin4-sulfate and chondroitin-6-sulfate were derived from whale cartilage, dermatan sulfate from swine skin, heparan sulfate from bovine liver and hyaluronic acid from human umbilical cord, respectively. After electrophoresis the strip was stained with alcian blue and scanned in an Atago Densitometer (Tokyo, Japan) in the normal way.

\section{RESULTS AND DISCUSSION}

Results of the protein, hexose and sialic acid analyses of Tamm-Horsfall glycoprotein in different fractions of its isolation procedure from the urine are summarized in Table 1. Actual figures for protein, hexose and sialic acid contents of Tamm-Horsfall glycoprotein from the original urine sample were $49 \pm 18.2 \mu \mathrm{g} / \mathrm{ml}$ (mean \pm standard deviations), $10 \pm 4.4 \mu \mathrm{g} / \mathrm{ml}$ and $2 \pm 0.6$ $\mu \mathrm{g} / \mathrm{ml}$, respectively. Values in the original urine sample are almost agreed with the data which were reported by Odin (1952) and Maxfield (1966). Two methods were adopted in order to separate the urine into fluid and cellular elements; either filtration with filter paper No. 2 or centrifugation at 1,500

TABLE 1

Distribution of Tamm-Horsfall glycoprotein in different urine fractions (\%)a

\begin{tabular}{l|c|c|c}
\hline \multicolumn{1}{c|}{ Fractions } & Protein & Hexose & Sialic acid \\
\hline Original urine & 100 & 100 & 100 \\
Filtered fraction & $32 \pm 16.0$ & $54 \pm 18.5$ & $22 \pm 4.0$ \\
Supernatant fraction & $78 \pm 19.4$ & $84 \pm 15.0$ & $86 \pm 8.0$ \\
\hline
\end{tabular}

a : Results given are averages of 5 samples. Figures following \pm sign are standard deviations of the mean.

r.p.m. for 15 minutes was carried out. Filtration procedure greatly reduced the Tamm-Horsfall glycoprotein content, while centrifugation had little effect on its content.

Glycosaminoglycan and glycopeptide were separated from urines both before and after isolation of Tamm-Horsfall glycoprotein, and then their contents were estimated. The average uronic acid, hexose and sialic acid contents of the glycosaminoglycan-glycopeptide preparations from both filtered fraction and supernatant fraction after centrifugation of the urine sample are in
Table 2. Actual figures for uronic acid, hexose and sialic acid contents of the glycosaminoglycan-glycopeptide preparation from the original urine sample before the isolation of Tamm-Horsfall glycoprotein were $2 \pm 1.0 \mu \mathrm{g} / \mathrm{ml}, 15 \pm 9.4$ $\mu \mathrm{g} / \mathrm{ml}$ and $2 \pm 0.7 \mu \mathrm{g} / \mathrm{ml}$, respectively. Values in the original urine are in general agreement with those of $\mathrm{Di}$ Ferrante (1967) and our earlier work (1978). It is of interest that either filtration procedure or centrifugation had no effect on the any of uronic acid, hexose or sialic acid content of the glycosaminoglycan-glycopeptide prepa- 
TABLE 2

Distribution of glycosaminoglycan and glycopeptide in different urine fractions before and after isolation of Tamm-Horsfall glycoprotein (\%) a

\begin{tabular}{l|c|c|c}
\hline \multicolumn{1}{c|}{ Fractions } & Uronic acid & Hexose & Sialic acid \\
\hline Before isolation of T-H glycoprotein & & & \\
$\quad$ Original urine & 100 & 100 & 100 \\
Filtered fraction & $100 \pm 30.1$ & $100 \pm 37.2$ & $100 \pm 24.5$ \\
$\quad$ Supernatant fraction & $100 \pm 24.2$ & $100 \pm 24.8$ & $100 \pm 23.2$ \\
After isolation of T-H glycoprotein & & & \\
Original urine & $76 \pm 30.1$ & $72 \pm 26.4$ & $94 \pm 34.4$ \\
Filtered fraction & $82 \pm 33.1$ & $72 \pm 27.9$ & $92 \pm 20.4$ \\
Supernatant fraction & $80 \pm 30.3$ & $70 \pm 20.0$ & $94 \pm 32.6$ \\
\hline
\end{tabular}

a : Results given are averages of 5 samples. Figures following \pm sign are standard deviations of the mean.

rations before isolation of Tamm-Horsfall glycoprotein. The average uronic acid, hexose and sialic acid content of the glycosaminoglycan-glycopeptide preparations after isolation of TammHorsfall glycoprotein are 6-30\% lower in all of the original, filtered and supernatant fractions than those before isolation of Tamm-Horsfall glycoprotein. But the reason is still unknown why the average uronic acid of the glycosaminoglycan - glycopeptide lpreparations after isolation of Tamm-Horsfall glycoprotein are lower than those before isolation of Tamm-Horsfall glycoprotein.

Cellulose acetate strip electrophoresis of urinary glycosaminoglycans in barbital buffer, as shown in Fig. 1, A, revealed the presence of three alcian blue positive bands in every fraction, numbered in order of decreasing electrophoretic mobility, band 1 , band 2 and band 3 . The band 1 was corresponded in mobility with chondroitin-4-sulfate, dermatan sulfate and chondroitin-6sulfate, the band 2 with heparan sulfate, and the band 3 with hyaluronic acid, respectively. Cellulose acetate strip electrophoretic patterns of urinary materials in calcium acetate are shown in Fig. 1, B. The preparations separated into two alcian blue positive bands in every case, numbered in order of decreasing electrophoretic mobility, band 1 and band 2 . The band 1 was corresponded in mobility with chondroitin-4sulfate and chondroitin-6-sulfate, and the band 2 with dermatan sulfate, heparan sulfate and hyaluronic acid, respectively. Any significant difference, however, was scarcely found among these different urine fractions either before or after isolation of TammHorsfall glycoprotein.

Aqueous suspensions of Tamm-Horsfall glycoprotein antigen from the original urine sample in 0.15 to $10 \mathrm{mg}$ per $\mathrm{ml}$ gave a single distinct zone of precipitation in the gel diffusion patterns with the rabbit anti Tamm-Horsfall glycoprotein. It made a similar single precipitin are corresponding to the region of albumin and $\alpha$-globulin in the immunoelectrophoresis. These data well agreed with the results of Keutel et al. (1961). Similar results were obtained by the similar studies on Tamm-Horsfall form both filtered fraction and supernatant fraction after centrifuga- 
(A) Barbital buffer

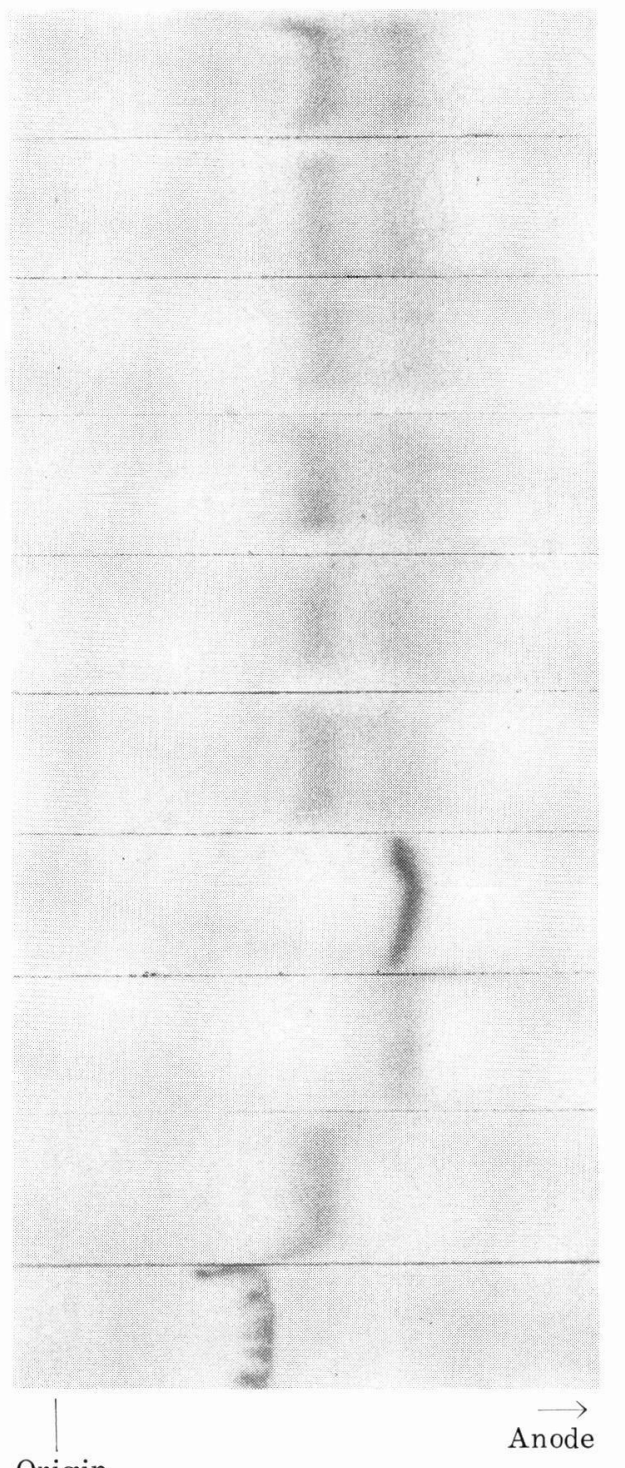

(B) Calcium acetate

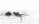

1

2

3

4

5

6

7

8

9

10

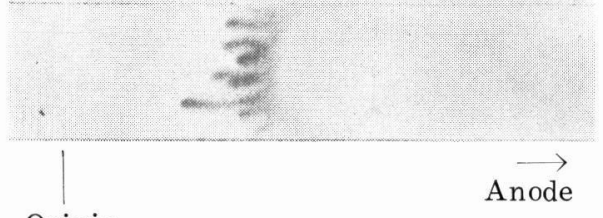

Origin

Fig. 1 Electrophoretic patterns of urinary glycosaminoglycans and marker samples. The material was run in both barbital buffer, $\mathrm{pH} 8.6$ ( $\mathrm{A}$, left side) and $0.3 \mathrm{M}$ calcium acetate, $\mathrm{pH} 7.5$ (B, right side). Electrophoretic migration is from left to right, as indicated by the arrow.

(1) original urine before isolation of $\mathrm{T}-\mathrm{H}$ glycoprotein,

(2) filtered fraction before isolation of $\mathrm{T}-\mathrm{H}$ glycoprotein,

(3) supernatant fraction before isolation of $\mathrm{T}-\mathrm{H}$ glycoprotein,

(4) original urine after isolation of $\mathrm{T}-\mathrm{H}$ glycoprotein,

(5) filtered fraction after isolation of $\mathrm{T}-\mathrm{H}$ glycoprotein,

(6) supernatant fraction after isolation of $\mathrm{T}-\mathrm{H}$ glycoprotein,

(7) a mixture of chondroitin-4-sulfate and -6-sulfate,

(8) dermatan sulfate, (9) heparan sulfate, (10) hyaluronic acid. 
tion of the urine samples, while lower antigenic titer was obtained from the filtered fraction than both the original urine sample and the supernatant fraction (Fig. 2 and 3).

It is necessary to separate and take off both cellular elements and urinary casts from the fluid of the urine in order to study urinary complex carbohydrate. Either filtration or centrifugation is usually adopted for this purpose, and so both methods were carried out and compared each other. Filtration procedure greatly reduced the TammHorsfall glycoprotein content, while centrifugation had little effect on its content, and furthermore either the former or the latter had no effect on the content of glycosaminoglycan-glycopeptide preparations. These differences are explainable on the basis of differences in both their molecular size and mo'ecular structure. Tamm-Horsfall glycoprotein is made up of filaments having a width of about $10 \mathrm{~nm}$ and an average length of about 2,500 nm (Porter and Tamm, 1954), while proteoglycan has a wide spread structure having an average width of $118 \mathrm{~nm}$ and a length of $308 \mathrm{~nm}$ (Yoneda, 1977).
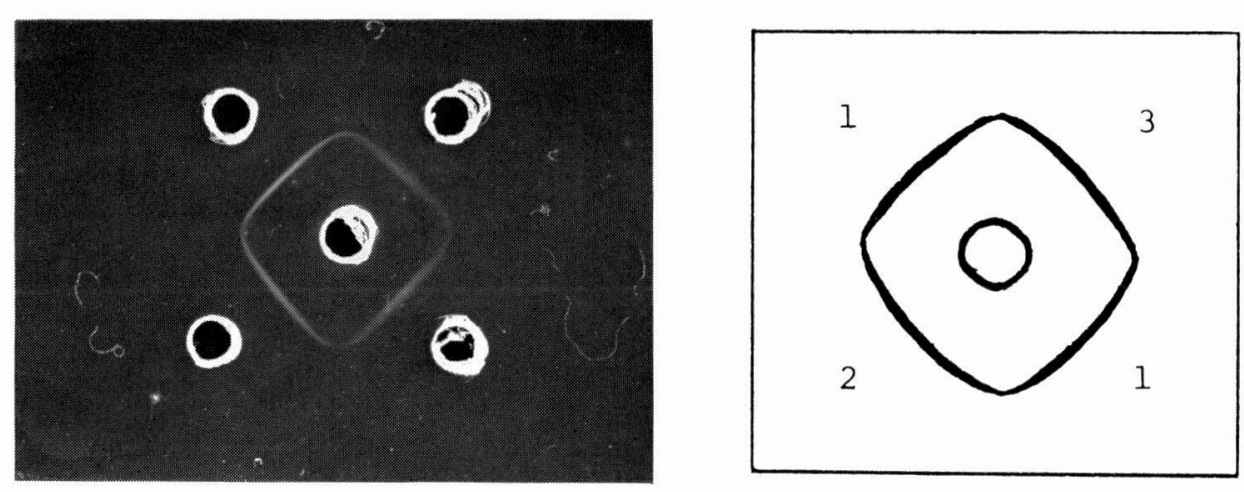

Fig. 2 Ouchterlony double diffusion tests.

(1) ; Tamm-Horsfall glycoprotein from the original urine sample,

(2) ; Tamm-Horsfall glycoprotein from the filtered fraction of the urine,

(3); Tamm-Horsfall glycoprotein from the supernatant fraction after centrifugation of the urine, Central well ; Anti-Tamm-Horsfall glycoprotein antibody (rabbit).

This work has been supported by the grant from the Graduates' Association of Kurume University.

\section{REFERENCES}

Di Ferrante, N.M. (1976). The measurement of urinary mucopolysaccharides. Analyt. Biochem., 21, 98-106.

Dische,Z. (1962). In "Methods in Carbohydrate Chemistry" (R. L. Whistler and M. L. Wolform, eds.), Vol. 1, pp. 477-500. Academic Press Inc., New York, New York.
Keutel, H. J., Heremans, J. and Vaerman, J. P. (1961). In "Protides of the Biological Fluids, 8 th Colloquium” (H. Peeters, ed.), pp. 480-484. Elsevier, Amdsterdam.

Lowry, O. H., Rosebrough, N. J., Farr, A. L. and RANDALl, R. J. (1951). Folin phenol reagent for the determination of protein. J. Biol. Chem., 193, 25.

Masuda, H., Shichijo,S., Imai,H., Takeuchi, M. and Shetlar, M. R. (1978). Urinary glycopeptides and glycosaminoglycans in children and adults as affected by age. Texas Rep. Biol. Med., (in press). 

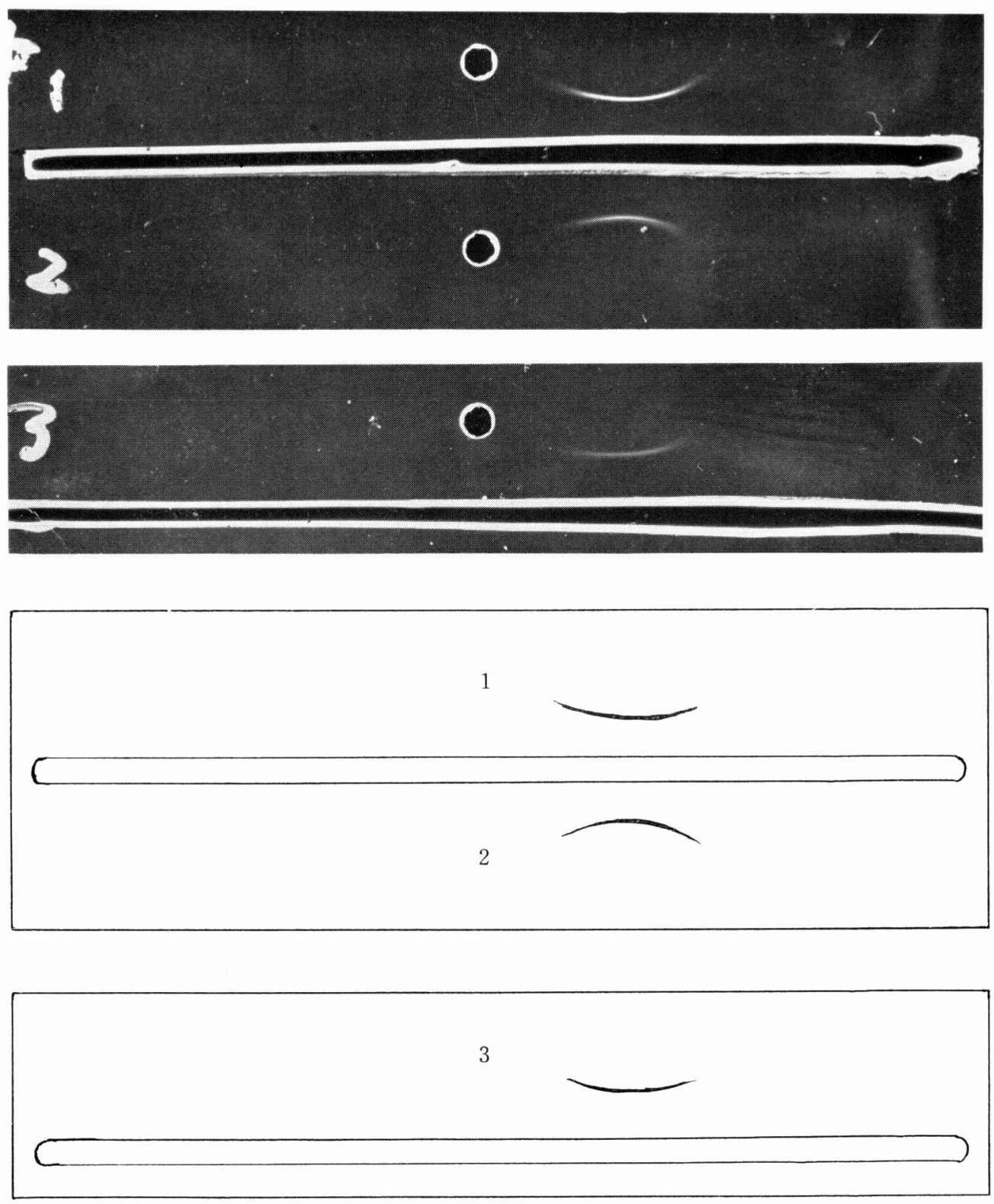

$(-)$

Fig. 3 Immunoelectrophoretic patterns.

(1) ; Tamm-Horsfall glycoprotein from the original urine sample,

(2) ; Tamm-Horsfall glycoprotein from the filtered fraction of the urine,

(3) ; Tamm-Horsfall glycoprotein from the supernatant fraction after centrifugation of the urine, Central toughs ; Anti-Tamm-Horsfall glycoprotein antibody (rabbit). 
MAXFIELD, M. (1966). In “Glycoproteins" (A. Gottschalk, ed.), pp. 446-457. Elsevier Publishing Co., New York.

Mukusick, V. A. (1972). In "Heritable disorders of connective tissue", 4 th ed., pp. 521-540. C. V. Mosby Co., St. Louis, Missouri.

Odin, L. (1952). Analysis of Tamm-Horsfall glycoprotein. Nature, 170, 662-663.

PorTer, K. R. and TAMM, I. (1954). Direct visualization of a mucoprotein component of urine. J. Biol. Chem, 212, 135.

SAITo, H. (1973). Urinary mucoproteins. 2, Clinical investigation in the diseases of the kidney and urinary tract. Jap. J. Urol, 63, 241.

Shiller, S., Slover, G. A. and Dolfman, A. (1961). A method for the separation of acid mucopolysaccharides; its application to the isolation of heparin from the skin of rats. J. Biol. Chem, 236, 983-990.

TAMM, I. and Horsfall, F. L. Jr. (1950). Characterization and separation of an inhibitor of viral hemmagglutination present in urine. Proc. Soc. Exptl. Biol. Med., 74, 108.

WINZLER, R. J. (1955 a). In "Methods of Biochemical Analysis" (D. Glick, eds.), II, pp. 279-285. Interscience Publishers, New York. WINZLER, R. J. (1955 b). In “ibid.", 298-306, ibid., New York.

YoNedA, M. (1977). Electron microscopic studies of proteoglycans from epiphyseal cartilage of suckling rat. Connective Tissue, 9, 1. 